\title{
Sexual dimorphism in echolocation pulse parameters of the CF-FM bat, Hipposideros pratti
}

\author{
Zi-Ying Fu', Xing-Yue Dai ${ }^{1}, \mathrm{Na} X \mathrm{X}^{1}$, Qing Shi', Gao-Jing Li', Bin Li ${ }^{1}$, Juan $\mathrm{Li}^{1}$, Jie Li', Jia Tang ${ }^{1}$, \\ Philip Hung-Sun Jen ${ }^{2,3,4^{*}}$ and Qi-Cai Chen ${ }^{1 *}$
}

\begin{abstract}
Background: Previous studies of sexual dimorphism in the echolocation pulses of the constant frequency-frequency modulating (CF-FM) bat have been mainly concentrated on the difference in the frequency of the CF component of the predominant second harmonic while neglected other pulse parameters. However, recent studies have shown that other pulse parameters of the predominant second harmonic are also biologically significant to the bat hunting. To complement and advance these studies, we have examined sexual dimorphism of multiple parameters (e.g., duration, frequency, bandwidth of the FM component, and repetition rate of emitted pulses) of the echolocation pulses of the CF-FM bat, Hipposideros pratti.
\end{abstract}

Results: Our studies of the predominant second harmonic show that on average the male bat has higher frequency of the CF component, wider FM bandwidth, and higher pulse repetition rate while the female bat has longer duration of the CF and FM components.

Conclusions: These observations suggest that bats may potentially use this sexual dimorphism in echolocation pulse parameters for social communication and species and sex identification.

Keywords: CF-FM bat; Echolocation pulse; Sexual dimorphism; Hipposideros pratti

\section{Background}

During hunting, insectivorous bats mainly emit either multiple frequency modulating (FM) or constant frequencyfrequency modulating (CF-FM) echolocation pulses and analyze the returning echoes to extract information about the target features (e.g., size, shape, texture, velocity, range etc.) (Griffin 1958). Based on the frequency content of these echolocation pulses, these bats have been conveniently called FM or CF-FM bats (Griffin 1958; Neuweiler 2003; Ulanovsky and Moss 2008). The echolocation pulses of these bats are typically multiple harmonics with the first one being the fundamental frequency. Also, the echolocation pulse energy is not evenly distributed among different harmonics. For example, the CF-FM bat emits a long CF component followed by a short FM component, and the

\footnotetext{
* Correspondence: jenp@missouri.edu; chenqc@mail.ccnu.edu.cn ${ }^{2}$ College of Special Education, Binzhou Medical College, Yantai, Shandong, China

${ }^{1}$ School of Life Sciences and Hubei Key Lab of Genetic Regulation and Integrative Biology, Central China Normal University, Wuhan 430079Hubei, China

Full list of author information is available at the end of the article
}

pulse energy is predominantly distributed in the second harmonic $\left(H_{2}\right)$ (i.e., predominant harmonic) (Griffin 1958; Jones and Teeling 2006; Neuweiler 2003; Suga 1990; Vanderelst et al. 2013). It has been proposed that the CF component is used for echo detection and evaluation of Doppler-shifted echo frequency while the FM component is effective for echo ranging and fine target feature analysis (Fitzpatrick et al. 1991; Jakobsen et al. 2013; Kober and Schnitzler 1990; O'Neill and Suga 1982; Schnitzler and Denzinger 2011; Simmons and Vernon 1971).

In addition to using the echolocation pulses for hunting, the bats also use these pulses as social signals for communication, individual recognition, threatening, and guidance (Möhres 1967; Knörnschild et al. 2012; Kazial et al. 2008; Yovel et al. 2009; Voigt-Heucke et al. 2010). Other studies also showed that the echolocation pulses could be used for identification of species (Russo et al. 2007; Schuchmann and Siemers 2010), age (Jones and Ransome 1993; Masters et al. 1995), and group affiliation (Jameson and Hare 2009; Masters et al. 1995). Presumably, these social interactions are based on the fact that 
there are variations on pulse parameters among individual bats and between female and male bats (sexual dimorphism).

Sexual dimorphism in the echolocation pulses has been reported for both FM and CF-FM bats (Feng et al. 2003; Grilliot et al. 2009; Jones et al. 1992; Jones et al. 1993; Kazial and Masters 2004; Knörnschild et al. 2012; Russo et al. 2001; Siemers et al. 2005; Suga et al. 1987). Interestingly, most of these studies have been devoted to examining sexual dimorphism of echolocation pulses in the CF-FM bat (Feng et al. 2003; Jones et al. 1992; Knörnschild et al. 2012; Russo et al. 2001; Siemers et al. 2005). This is perhaps due to the fact that this bat performs unique Doppler-shifted compensation behavior during which the bat appropriately adjusts the frequency of the emitted pulse to compensate for the Dopplershifted frequency of the returning echo. Furthermore, most of these studies mainly examined the sexual dimorphism of the frequency and duration of the $\mathrm{H}_{2}$ of the echolocation pulses (conveniently referred as CF hereafter) while neglected other pulse parameters. However, recent studies have shown that the FM component of the CF-FM pulse not only plays an essential role in shaping the response properties of central auditory neurons (the inferior colliculus) (Fu et al. 2010) but also facilitates the selectivity of these neurons in duration (Luo et al. 2008; Macías et al. 2013), amplitude (Fu et al. 2011), and the recovery cycle (Tang et al. 2011). These studies suggest that the CF-FM bat may utilize the information provided by the variation in the $\mathrm{CF}$ and $\mathrm{FM}$ components of the echolocation pulses throughout the entire course of hunting for successful prey capture. These studies also implicate that bats may utilize the variation in the FM components and other pulse parameters if any for social interactions.

In this study, we studied the sexual dimorphism of the echolocation pulses by analyzing multiple pulse parameters (e.g., duration, frequency, bandwidth of the FM component, and repetition rate of echolocation pulses) of the predominant $H_{2}$ which has been shown to be essential for successful echolocation in behavioral and neurophysiological studies (Neuweiler et al. 1987; O'Neill and Suga 1982; Schnitzler and Henson 1980; Schnitzler and Denzinger 2011; Suga et al. 1987; Suga 1990). We report here that the male bat has higher CF, wider FM bandwidth, and higher pulse repetition rate (PRR) while the female bat has longer duration of both CF and FM components (conveniently referred as CF duration and FM duration hereafter).

\section{Methods}

Animals and morphological studies

In this study, we used 34 adult Hipposideros pratti ( $H$. Pratti) (13 males and 21 females, 34.5-48.1 g body weight (b. w.)) caught from a cave near Xianning city of Hubei providence of People's Republic of China in May of 2013 and 2014. They were caught from the same colony by means of mist net with the permission of Central China Normal University. These bats were housed in groups (three to five bats each group) in a cage within a temperature- and humidity-controlled animal room and were given larvae of Tenebrio molitor plus vitamin-mineralenriched water ad libitum. The bats were trained to eat larvae and drink water for about 1 week. The age category of the bat was determined on the basis of whether phalangeal epiphyses were fused with the diaphysis, and its sex was assessed by inspecting the genitalia (Racey 1988). Each bat was weighed to the nearest $1 \mathrm{~g}$ with a Pesola balance, and the length of its right forearms was measured to the nearest $0.1 \mathrm{~mm}$ with dial calipers. This study was conducted with the approval of the Institutional Animal Care and Use Committee of Central China Normal University, Wuhan, Hubei, People's Republic of China.

\section{Recordings of echolocation pulses}

The recording of the echolocation pulses was made a few days after each bat was acclimated to the laboratory condition. The recording of the echolocation pulses was made at about 7 p.m. in May of 2013 and 2014. During recording, each bat was hanging on the ceiling of the experimental anechoic room and the recording microphone was placed $1 \mathrm{~m}$ below. The echolocation pulses were recorded only from each sedentary bat in real time with a handheld ultrasound detector (Petterson D1000X; Pettersson Elektronik $A B$, Uppsala, Sweden) before being fed into a notebook computer. Each bat was recorded 1-3 times and each time lasted 3-5 s.

\section{Pulse analysis}

The echolocation pulses were analyzed using the software BatSound pro 3.31b (Pettersson Elektronik AB, Uppsala, Sweden), with a fast Fourier transformation (FFT) size of 1024 points and a Hanning window using a cursor and visual determination on a screen. We only analyzed high-quality pulses with good signal-to-noise ratio. To characterize each echolocation pulse, we analyzed the CF, FM bandwidth, and durations of both $\mathrm{CF}$ and FM components of the $H_{2}$ as well as the PRR. The time parameters were taken from amplitude waveforms, and frequency parameters were taken from spectrograms. In the present study, when we calculated the CF difference between the recorded pulses from male and female bats, we firstly calculated the CF average of all pulses recorded from a same male or female bat as a male or female individual, i.e., as a sampling unit. Then, we put each $\mathrm{CF}$ average from each male or female bat 
together and calculated the total mean CF value from all male and female individuals.

\section{Statistical analysis}

All data obtained were processed and plotted using Sigmaplot 2000 (Systat Software, San Jose, CA, USA). They were then quantitatively examined and statistically compared using SPSS 13.0 (Student's $t$ test at $p<0.05$ ) (SPSS, Chicago, IL, USA).

\section{Results}

\section{The forearm and body weight of Hipposideros pratti}

The measurement of the forearm and body weight of these 34 bats is shown in Table 1. It is clear that there are differences between male and female bats and the former have longer average forearm length but slightly lighter body weight than the latter. However, these differences are not statistically significant $(p>0.05)$.

\section{Echolocation pulses of Hipposideros pratti}

In this study, 1574 individual echolocation pulses were recorded from these 34 bats ranging from 24 to 76 pulses (average 46 pulses) from each bat. Fig. 1 shows the sonogram and power spectrum of a representative echolocation pulse. The echolocation pulse consists of 4 harmonics with the first harmonic being the fundamental frequency. Each of these harmonics starts with a long CF component followed by a short FM component. The analysis of the power spectrum of the pulse clearly shows that the second harmonic $\left(H_{2}\right)$ is the predominant one that contains the highest energy. This is evident by the fact that the $\mathrm{H}_{2}$ has the darkest band in Fig. 1a and the highest peak in Fig. 1b. The measurements of CF, FM bandwidth, CF duration, FM duration, and PRR of the $H_{2}$ of echolocation pulses from all these 34 bats are shown in Table 2. Although there are differences among these multiple pulse parameters, the $H_{2}$ of this bat has an average of about 6.3-ms CF component at $60 \mathrm{kHz}$ followed by $2.5-\mathrm{ms}$ FM component with $10-\mathrm{kHz}$ bandwidth. Each

Table 1 Measurements of the forearm and body weight of 13 male and 21 female Hipposideros pratti

\begin{tabular}{llll}
\hline & \multicolumn{2}{c}{ Sex (sample size $)$} & Student's $t$ test $p$ \\
\cline { 2 - 3 } & Male $(n=13)$ & Female $(n=21)$ & \\
\hline Forearm length $(\mathrm{mm})$ & & & \\
Range & $82.00-89.06$ & $81.00-87.54$ & $>0.05$ \\
Mean \pm sd & $86.41 \pm 2.36$ & $85.21 \pm 2.05$ & \\
Body weight $(\mathrm{g})$ & & & \\
Range & $34.6-48.1$ & $34.5-47.9$ & $>0.05$ \\
Mean \pm sd & $41.2 \pm 4.6$ & $39.7 \pm 4.1$ & \\
\hline
\end{tabular}

$n$ number of bats hanging bat emitted its echolocation pulses at a rate about 14 pulses per second.

\section{Sexual dimorphism of echolocation pulse parameters in Hipposideros pratti}

We examined the sexual dimorphism of echolocation pulses in this bat species by separately plotting the distribution of average value of these multiple parameters of echolocation pulses obtained from each male and female bat. As shown in Fig. 2, the predominant CF of the male bat was between 58.8 and $60.5 \mathrm{kHz}$ with most above $59.5 \mathrm{kHz}(61.5 \%$, Fig. 2(A-1)) while that of the female bat was between 58.0 and $59.9 \mathrm{kHz}$ with most below $59.5 \mathrm{kHz}(57.1 \%$, Fig. 2(A-2)). This sexual dimorphic difference in CF $(0.63 \mathrm{kHz})$ was statistically significant $(59.91 \pm 0.43$ vs. $59.28 \pm 0.56 \mathrm{kHz}, n=13$ for male, $n=21$ for female, $T=3.509, p<0.01$, $t$ test, Fig. 2(A-3)). The FM bandwidth of the male bat ranged from 9.1 to $12.7 \mathrm{kHz}$ with most above $9.5 \mathrm{kHz}$ $(69.28 \%$, Fig. 2(B-1)) while that of the female bat ranged from 8.1 to $10.9 \mathrm{kHz}$ with most below $9.5 \mathrm{kHz}$ (61.9 \%, Fig. 2(B-2)). This sexual dimorphic difference in FM bandwidth was also statistically significant $(10.08 \pm 1.01$ vs. $9.38 \pm 0.81 \mathrm{kHz}, n=13$ for male, $n=21$ for female, $T=2.239, p<0.05, t$ test, Fig. 2(B-3)). The PRR of the male bat ranged between 4.0 and $31.0 \mathrm{~Hz}$ with most (61.5 \%) higher than $15.0 \mathrm{~Hz}$ (Fig. 2(C-1)). Different from this observation, the PRR of the female bat ranged between 2.7 and $27.0 \mathrm{~Hz}$ and most (85.7\%) of them were below $15 \mathrm{~Hz}$ (Fig. 2(C-2)). A statistical analysis revealed a significant sexual dimorphic difference in the average PPR of echolocation pulse of this bat species $(16.3 \pm 7.8$ vs. $10.9 \pm 6.2 \mathrm{~Hz}, n=13$ for male, $n=21$ for female, $T=2.288, p<0.05, t$ test, Fig. 2(C-3)).

The distribution of the averaged CF and FM duration of the predominant $\mathrm{H}_{2}$ component of the echolocation pulses of each male and female $H$. pratti is shown in Fig. 3. The CF duration of the predominant $\mathrm{H}_{2}$ of the male bat was between 4.2 and $8.8 \mathrm{~ms}$ with more than half $(69.2 \%)$ shorter than $6 \mathrm{~ms}$ (Fig. 3(A-1)). In contrast, the CF duration of the predominant $\mathrm{H}_{2}$ of the female bat was between 5.4 and $10.9 \mathrm{~ms}$ with most $(81.0 \%)$ longer than $6.0 \mathrm{~ms}$ (Fig. 3(A-2)). This sexual dimorphic difference in the CF duration of the predominant $H_{2}$ was statistically significant $(5.7 \pm 1.1$ vs. $7.3 \pm$ $1.6 \mathrm{~ms}, n=13$ for male, $n=21$ for female, $T=-3.066, p<$ $0.05, t$ test, Fig. 3(A-3)). The FM duration of the predominant $\mathrm{H}_{2}$ of the male bat was between 1.3 and $2.9 \mathrm{~ms}$ with $61.5 \%$ shorter $2.5 \mathrm{~ms}$ while that of the female bat ranged from 2.0 to $3.2 \mathrm{~ms}$ with most (71.4 \%) longer than $2.5 \mathrm{~ms}$ (Fig. 3(B-1) vs. (B-2)). The sexual dimorphic difference in this measurement was also statistically significant $(2.4 \pm 0.3$ vs. $2.7 \pm 0.4 \mathrm{~ms}, n=13$ for male, $n=21$ for female, $T=-3.058, \mathrm{p}<0.05, t$ test, Fig. 3(B-3)). 

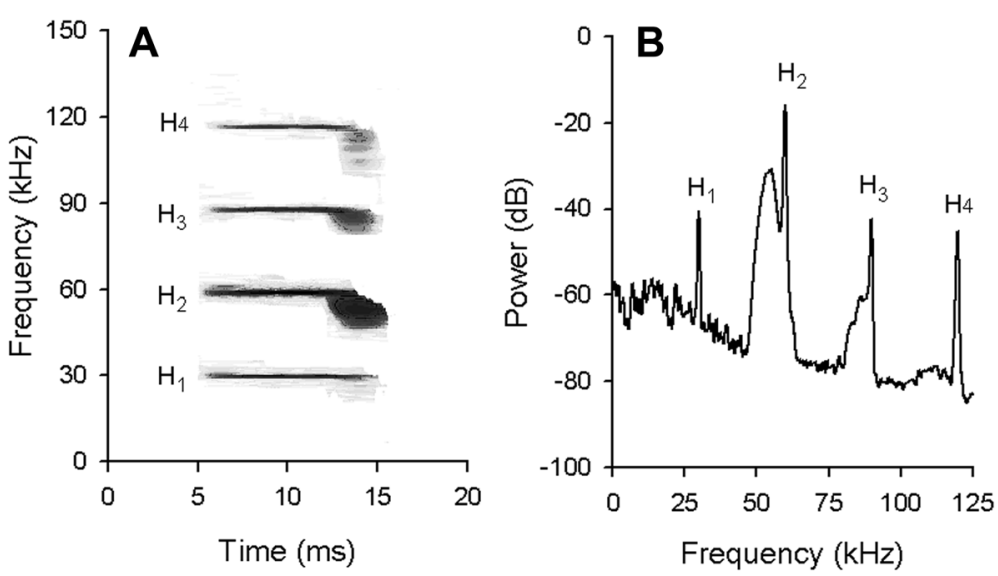

Fig. 1 The sonogram (a) and power spectrum (b) of a representative echolocation pulse emitted by a hanging Hipposideros pratti. The CF (kHz), FM bandwidth ( $\mathrm{kHz}$ ), CF duration (ms), and FM duration (ms) of this echolocation pulse were 60.0, 12.8, 7.5, and 2.7. Clearly, the second harmonic is the predominant one that contains the highest energy

\section{Discussion}

The forearm length, body weight, and echolocation pulses of Hipposideros pratti

The forearm length, body weight, and the predominant CF of $H$. pratti measured in the present study (Fig. 1, Tables 1 and 2, bats caught in Hubei province) were similar to the measurements of the same bat species reported in a previous study (bats caught in Fujian, Guangxi, and Henan provinces, Zhang et al. 2009) but different from that reported in another study (bats caught in Guizhou province, Chen et al. 2002). The difference in these measurements of the same bat species may be due to geographic difference. Regardless of these different reports, our present study did not find any sexual dimorphism in the forearm length and body weight of $H$. pratti (Table 1 ).

\section{Sexual dimorphism of multiple parameters of the predominant $\mathrm{H}_{2}$}

In this study, we observed the sexual dimorphism of multiple parameters in the predominant $\mathrm{H}_{2}$ of the echolocation pulses of $H$. pratti (Figs. 2 and 3, Table 2). On average, the male bat has higher predominant CF, wider FM bandwidth, and higher PRR while the female bat has longer duration of the CF and FM components. This observed sexual dimorphism in the multiple pulse parameters in this bat

Table 2 Parameters of the predominant second harmonic $\left(\mathrm{H}_{2}\right)$ of the echolocation pulses emitted by Hipposideros pratti

\begin{tabular}{llll}
\hline Parameters & Mean \pm sd & Median & Min-max \\
\hline CF frequency $(\mathrm{kHz})$ & $59.5 \pm 0.6$ & 59.7 & $58.0-60.5$ \\
FM bandwidth $(\mathrm{kHz})$ & $9.6 \pm 0.9$ & 9.5 & $8.1-12.7$ \\
CF duration $(\mathrm{ms})$ & $6.7 \pm 1.6$ & 5.9 & $4.2-10.9$ \\
FM duration $(\mathrm{ms})$ & $2.6 \pm 0.4$ & 2.6 & $1.6-3.2$ \\
Pulse repetition rate $(\mathrm{Hz})$ & $13.0 \pm 7.2$ & 11.5 & $2.7-31.0$ \\
\hline
\end{tabular}

CF constant frequency, FM frequency modulation species is not likely related to the difference in the forearm length and body weight since these two latter physical parameters were not significantly different between the male and female bats (Table 1).

Previous studies on sexual dimorphism of echolocation pulses of the CF-FM bats were mainly focused on the predominant CF (Jones et al. 1992, 1993; Knörnschild et al. 2012; Russo et al. 2001; Feng et al. 2003; Siemers et al. 2005; Suga et al. 1987). For comparison, data on the sexual dimorphism of the predominant CF in different CF-FM bats examined in these previous and the present studies are assembled in Table 3. It is clear that significant sexual dimorphism in the predominant CF is observed in all but two bat species (e.g., Rhinolophinae euryale and Rhinolophinae mehelyi, Russo et al. 2001). It is also clear that the predominant CF of the same bat species (Rhinolophinae euryale) varies with geographic location (Russo et al. 2001; Siemers et al. 2005). This finding is similar to a recent report (Jiang et al. 2010).

As shown in Table 3, sexual dimorphism in the predominant CF varies with bat species. There is no consistent pattern in the sexual dimorphism in the predominant CF difference in these bat species. For example, in Rhinolophidae, the CF was higher in the female than in the male bat but the opposite was observed in other studies. This is also true in the predominant CF in all Hipposiderinae bat species studied. On the one hand, these different observations on the sexual dimorphism of the predominant CF might be due to species-specific difference, different methods of analysis of the echolocation pulses, or due to the different ways that the echolocation pulses were recorded (Luo et al. 2007). On the other hand, it has been suggested that these different observations might be due to geographic location, different dimensions of vocal cord, and sex hormone level (Neuweiler et al. 1987; Russo et al. 2001). While the largest sexual dimorphic CF difference is 


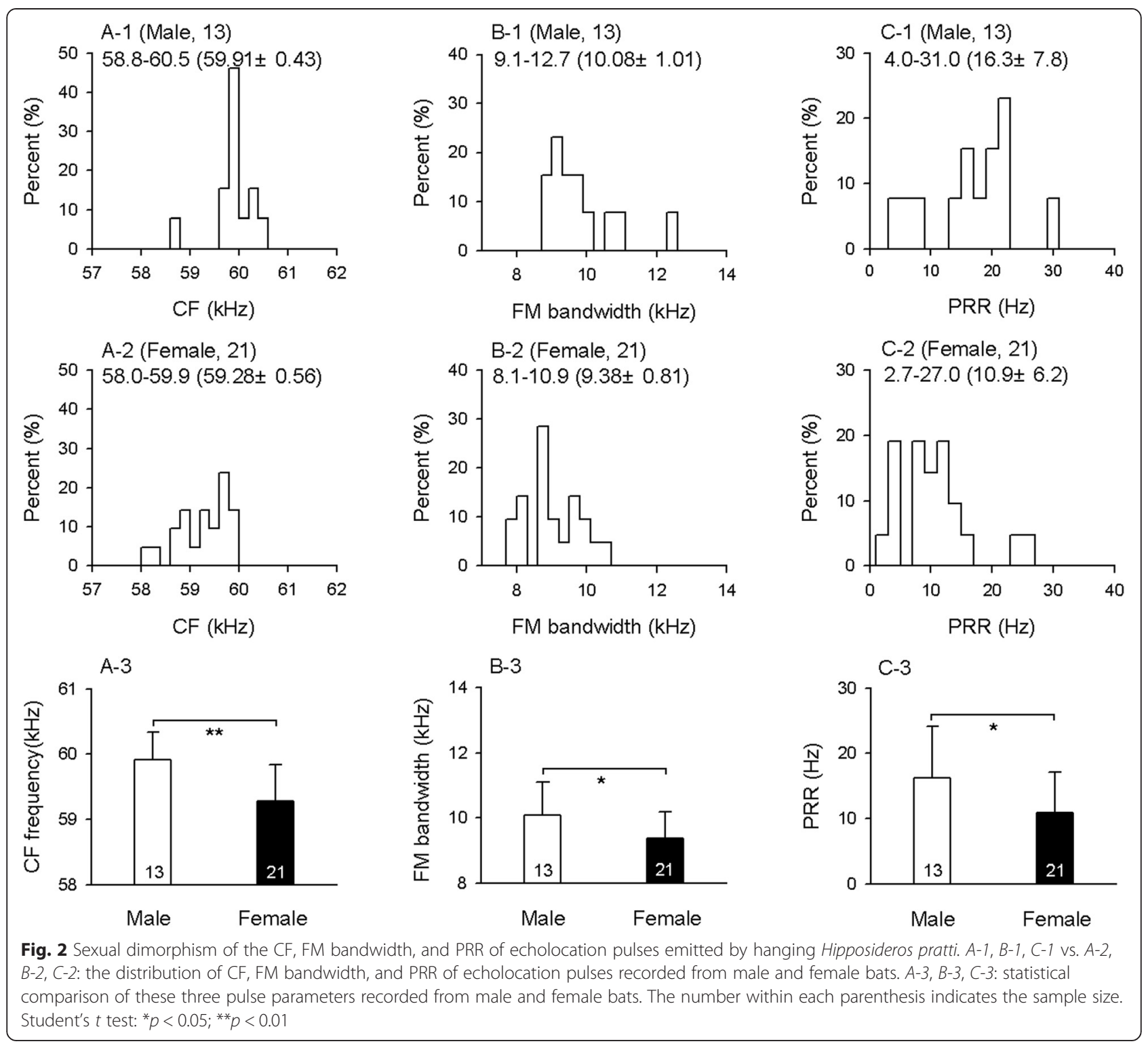

$10.49 \mathrm{kHz}$ in Hipposideros caffer (Jones et al. 1993), the smallest sexual dimorphic CF difference is only $0.8 \mathrm{kHz}$ in Rhinolophinae euryale (Siemers et al. 2005). The CF difference of $0.63 \mathrm{kHz}$ between male and female bats (H. pratti) in the present study is comparable and significant (Fig. 2(A-1) vs. (A-2)). Because the bats were from a same colony of the same cave, there were the possible relativity among the individuals and the possible similarity among the recorded pulses.

In addition to examining sexual dimorphism in the predominant $\mathrm{CF}$, we also observed that the FM bandwidth was significantly wider and the PRR was significantly higher in male than in female $H$. pratti (Fig. 2). On the contrary, the duration of both CF and FM was significantly longer for female than for male bat (Fig. 3). Whether these multi-parametric dimorphic differences are due to the length and width of vocal cord between the male and female bats remains to be studied. If they were different significantly, combined with the fact that the prey detection may be a primary selective force influencing the echolocation features of bats, it might help us to realize our data. In the case of female bats, they have lower CF than male bats due to their laryngeal structure; to increase pulse intensity, they may shorten the bandwidth (Lazure and Fenton 2011); and to get adequate information, they may increase the duration, simultaneously leading to the PRR reduction considering that low-duty-cycle bats separate pulse and echo in time (Lazure and Fenton 2011; Ho et al. 2013). And the echolocation features of male bats are opposite to the female bats, which may also be beneficial for them to make best use of the predation space and distinct each other and resist jamming. 

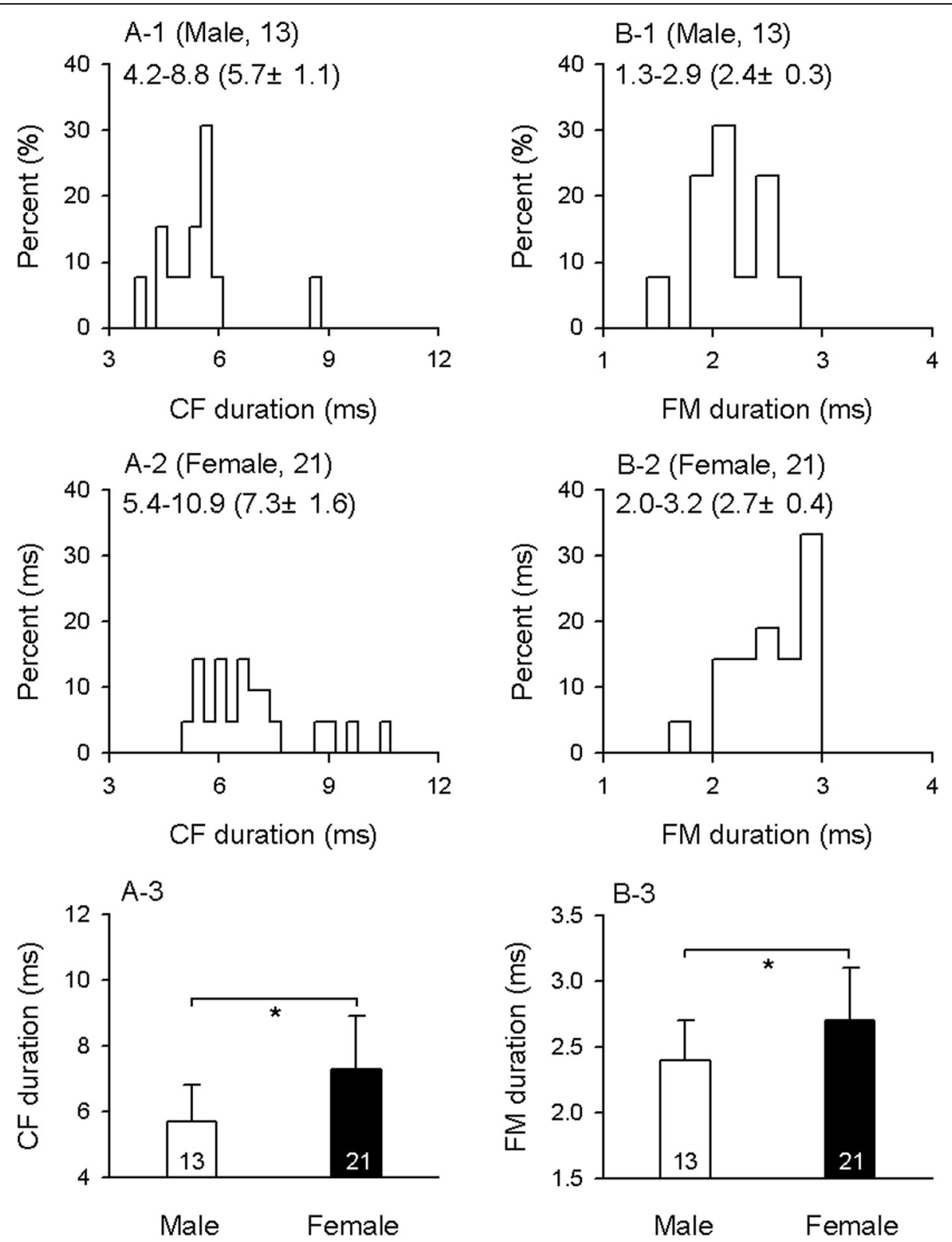

Fig. 3 Sexual dimorphism of the CF and FM duration of echolocation pulses emitted by hanging Hipposideros pratti. A-1, B-1, C-1 vs. A-2, B-2, C-2: the distribution of CF and FM duration of echolocation pulses recorded from male and female bats. $A-3, B-3, C-3$ : statistical comparison of these two temporal parameters of echolocation pulses recorded from male and female bats (see Fig. 2 for legends)

Possible biological significance of this study

Although there have been studies on sexual dimorphism of bat communication sounds (Kanwal et al. 1994; Liu et al. 2013; Ma et al. 2006), many studies have been mainly focused on the sexual dimorphism of orientation pulses in the CF-FM bat by analyzing the predominant CF (Jones et al. 1992, 1993; Knörnschild et al. 2012; Russo et al. 2001; Feng et al. 2003; Siemers et al. 2005; Suga et al. 1987). In the present study, we have clearly shown that sexual dimorphism of echolocation pulses in the CF-FM bat can also be found in other multiple pulse parameters. For this reason, the present study not only complements but also advances all previous studies with more detailed analysis of sexual dimorphic differences in the echolocation pulses of the CF-FM bat.

While the pulse energy is unevenly distributed on the multiple harmonics of the echolocation pulses of the CF-FM bat, the predominant $H_{2}$ has been shown to be essential for successful echolocation in behavioral and neurophysiological studies (Neuweiler et al. 1987; O’Neill and Suga 1982; Schnitzler and Henson 1980; Schnitzler and Denzinger 2011; Suga et al. 1987; Suga 1990). Because 
Table 3 Sexual dimorphism in the CF in echolocation pulses of CF-FM bats

\begin{tabular}{|c|c|c|c|}
\hline Species & Male & Female & Significances \\
\hline \multicolumn{4}{|l|}{ Rhinolophidae } \\
\hline \multicolumn{4}{|l|}{ Rhinolophinae euryale } \\
\hline South Bulgaria (Siemers et al. 2005) & $105.56 \pm 0.43$ & $106.63 \pm 0.62$ & s \\
\hline North Bulgaria (Siemers et al. 2005) & $106.21 \pm 0.84$ & $105.83 \pm 0.41$ & s \\
\hline Italy (Russo et al. 2001) & $104.32 \pm 0.39$ & $104.35 \pm 0.33$ & n. s. \\
\hline Rhinolophinae cornutus (Feng et al. 2003) & $103.13 \pm 1.95$ & $106.71 \pm 2.30$ & s \\
\hline Rhinolophinae mehelyi (Russo et al. 2001) & $108.10 \pm 0.88$ & $107.48 \pm 0.94$ & n. s. \\
\hline \multicolumn{4}{|l|}{ Hipposiderinae } \\
\hline Hipposideros rubber (Jones et al. 1993) & $134.13 \pm 1.84$ & $131.85 \pm 0.99$ & s \\
\hline Hipposideros caffer (Jones et al. 1993) & $138.83 \pm 8.25$ & $149.32 \pm 4.35$ & s \\
\hline Asellia tridens (Jones et al. 1993) & $116.37 \pm 1.42$ & $118.77 \pm 1.59$ & s \\
\hline Hipposideros armiger (Feng et al. 2003) & $77.52 \pm 0.11$ & $76.27 \pm 0.78$ & s \\
\hline Aselliscus wheeleri (Feng et al. 2003) & $125.53 \pm 0.22$ & $123.33 \pm 1.59$ & s \\
\hline Hipposideros pratti (present study) & $59.91 \pm 0.43$ & $59.28 \pm 0.56$ & s \\
\hline \multicolumn{4}{|l|}{ Mormoopidae } \\
\hline Pteronotus parnellii (Suga et al. 1987) & $61.25 \pm 0.534$ & $62.29 \pm 0.539$ & s \\
\hline
\end{tabular}

Data displayed in the table are expressed as the mean \pm standard deviation. Recording condition of the bat was hand-held (Jones et al. 1993; Russo et al. 2001; Siemers et al. 2005), flying (Feng et al. 2003), or sedentary (Suga et al. 1987; present study)

$s$ significant difference, $n$. s. not significant difference

of the time constraint, as the first step, we studied the sexual dimorphism in the multiple pulse parameters of the predominant $\mathrm{H}_{2}$ of echolocation pulses emitted from sedentary bats mimicking the hanging bats in the roosting cave. An analysis of these multiple parameters of echolocation pulses emitted by flying bat during hunting or foraging should be conducted to further study the sexual dimorphism in echolocation pulse parameters of this bat species. Conceivably, sexual dimorphism in the predominant $H_{2}$ of echolocation pulse parameters may also be observed under field study.

During hunting, the bat systematically varies the echolocation pulses in multiple parametric domains to obtain as much information as possible from the returning echoes for successful prey capture and avoidance of obstacles (Griffin 1958; Jen and Kamada 1982; Schnitzler and Henson 1980). It has been shown that the dynamic variation in multiple pulse parameters throughout the prey approach sequence improves the response sensitivity of bat auditory neurons in multiple parametric domains and likely increases the success of hunting (Jen et al. 2001; Wu and Jen 2006, 2008). As mentioned in the "Background" section, many studies have shown that the bats use echolocation pulses for prey capture as well as for social signals for communication, individual recognition, threatening, and guidance (Jameson and Hare 2009; Jones and Ransome 1993; Kazial et al. 2008; Knörnschild et al. 2012; Masters et al. 1995; Möhres
1967; Russo et al. 2007; Schuchmann and Siemers 2010; Voigt-Heucke et al. 2010; Yovel et al. 2009). If our observed sexual dimorphism of echolocation pulses also occurs in nature, then the bats may potentially use this sexual dimorphism cues in pulse parameters for social communication and species and sex identification.

\section{Conclusions}

The present studies examined the sexual dimorphism in echolocation pulse parameters of the CF-FM bat, $H$. pratti. The data showed that on average the male bat has higher predominant CF, wider FM bandwidth, and higher pulse repetition rate while the female bat has longer duration of the CF and FM components. These observations suggest that bats may potentially use this sexual dimorphism in echolocation pulse parameters for social communication and species and sex identification.

\section{Abbreviations}

CF: constant frequency; FM: frequency modulating; $\mathrm{H}$ : harmonic; PRR: pulse repetition rate.

\section{Competing interests}

The authors declare that they have no competing interests.

\section{Authors' contributions}

Q-CC and Z-YF conceived and designed the experiments. Z-YF, X-YD, NX, QS, G-JL, BL, JuL, JiL, and JT performed the experiments. Z-YF, X-YD, NX, QS, G-JL, BL, JuL, JiL, JT, PH-SJ, and Q-CC analyzed the data. Q-CC contributed the reagents/materials/analysis tools. Z-YF, PH-SJ, and Q-CC wrote the paper. All authors read and approved the final manuscript. 


\section{Acknowledgements}

We thank the anonymous reviewers for their helpful comments on our earlier manuscript. The work was supported by grants (\#31200832, \#31070971) from the Natural Science Foundation of China and the Fundamental Research Funds of Central China Normal University (CCNU13A05027).

\section{Author details \\ 'School of Life Sciences and Hubei Key Lab of Genetic Regulation and Integrative Biology, Central China Normal University, Wuhan 430079Hubei, China. ${ }^{2}$ College of Special Education, Binzhou Medical College, Yantai, Shandong, China. ${ }^{3}$ Department of Life Science, National Taiwan Normal University, Taipei, China. ${ }^{4}$ Division of Biological Sciences, University of Missouri-Columbia, Columbia, MO 65211, USA.}

Received: 16 July 2014 Accepted: 6 May 2015

\section{Published online: 24 May 2015}

\section{References}

Chen M, Feng J, Li ZX, Zhou J, Zhao HH, Zhang SY, Sheng LX (2002) Echolocation sound waves, morphological features and foraging strategies in Hipposideros pratti. Chin J Appl Ecol 13:1629-1632 (in Chinese)

Feng J, Chen M, Liu Y, Li ZX, Zhang XC, Zhou J, Zhang SY (2003) Sexual differences in morphology and echolocation calls in five Chinese bat species. Acta Zool Sin 49:742-747 (in Chinese with English abstract)

Fitzpatrick DC, Suga N, Misawa H (1991) Are the initial frequency-modulated components of the mustached bat's biosonar pulses important for ranging? J Neurophysiol 66:1951-1964

Fu ZY, Tang J, Jen PHS, Chen QC (2010) The auditory response properties of single-on and double-on responders in the inferior colliculus of the leafnosed bat, Hipposideros armiger. Brain Res 1306:39-52

Fu ZY, Tang J, Li Y, Zeng H, Chen QC (2011) Frequency-modulation component of the mimic echolocation sound can increase the sensitivity of inferior collicular neurons to sound amplitude in the leaf-nosed bat, Hipposideros armiger. Zool Stud 50:537-545

Griffin DR (1958) Listening in the dark. Yale University Press, New Haven

Grilliot ME, Burnett SC, Mendoca MT (2009) Sexual dimorphism in big brown bat (Eptesicus fuscus) ultrasonic vocalizations is context dependent. J Mammal 90:203-209

Ho YY, Fang YP, Chou CH, Cheng HC, Chang HW (2013) High duty cycle to low duty cycle: echolocation behaviour of the hipposiderid bat Coelops frithii. PLoS One 8, e62938

Jakobsen L, Brinkløv S, Surlykke A (2013) Intensity and directionality of bat echolocation signals. Front Physiol 4:89

Jameson JW, Hare JF (2009) Group-specific signatures in the echolocation calls of female little brown bats (Myotis lucifugus) are not an artefact of clutter at the roost entrance. Acta Chiropterol 11:163-172

Jen PHS, Kamada T (1982) Analysis of orientation signals emitted by the CF-FM bat, Pteronotus p. parnellii and the FM bat, Eptesicus fuscus during avoidance of moving and stationary obstacles. J Comp Physiol A 148:389-398

Jen PHS, Zhou XM, Wu CH (2001) Temporally patterned sound pulse trains affect intensity and frequency sensitivity of inferior collicular neurons of the big brown bat, Eptesicus fuscus. J Comp Physiol A 187:605-616

Jiang T, Metzner W, You YY, Liu S, Lu GJ, Li S, Wang L, Feng J (2010) Variation in the resting frequency of Rhinolophus pusillus in Mainland China: effect of climate and implications for conservation. J Acoust Soc Am 128:2204-2211

Jones G, Ransome RD (1993) Echolocation calls of bats are influenced by maternal effects and change over a lifetime. Proc Biol Sci 252:125-128

Jones G, Teeling EC (2006) The evolution of echolocation in bats. Trends Ecol Evol 21:149-156

Jones G, Gordon T, Nightingale J (1992) Sex and age differences in the echolocation calls of the horseshoe bat, Rhinolophus hipposideros. Mammalia 56:189-193

Jones G, Morton M, Hughes PM, Budden RM (1993) Echolocation flight morphology and foraging strategies of some West African hipposiderid bats. J Zool 230:385-400

Kanwal JS, Matsumura S, Ohlemiller K, Suga N (1994) Analysis of acoustic elements and syntax in communication sounds emitted by mustached bats. J Acoust Soc Am 96:1229-1254

Kazial KA, Masters M (2004) Female big brown bats, Eptesicus fuscus, recognize sex from a caller's echolocation signals. Anim Behav 67:855-863
Kazial KA, Kenny TL, Burnett SC (2008) Little brown bats (Myotis lucifugus) recognize individual identity of conspecifics using sonar calls. Ethology 114:469-478

Knörnschild M, Jung K, Nagy M, Metz M, Kalko E (2012) Bat echolocation calls facilitate social communication. Proc Biol Sci 279:4827-4835

Kober R, Schnitzler HU (1990) Information in sonar echoes of fluttering insects available for echolocating bats. J Acoust Soc Am 87:882-896

Lazure L, Fenton MB (2011) High duty cycle echolocation and prey detection by bats. J Exp Biol 214(Pt 7):1131-1137

Liu Y, Metzner W, Feng J (2013) Vocalization during copulation behavior in greater horseshoe bats, Rhinolophus ferrumequinum. Chin Sci Bul 58:2179-2184

Luo F, Ma J, Li AA, Wu FJ, Chen QC, Zhang SY (2007) Echolocation calls and neurophysiological correlations with auditory response properties in the inferior colliculus of Pipistrellus abramus (Microchiroptera: Vespertilionidae). Zool Stud 46:622-630

Luo F, Metzner W, Wu FJ, Zhang SY, Chen QC (2008) Duration-sensitive neurons in the inferior colliculus of horseshoe bats: adaptations for using CF-FM echolocation pulses. J Neurophysiol 99:284-296

Ma J, Kobayasi K, Zhang S, Metzner W (2006) Vocal communication in adult greater horseshoe bats, Rhinolophus ferrumequinum. J Comp Physiol A 192:535-550

Macías S, Hechavarría JC, Kossl M, Mora EC (2013) Neurons in the inferior colliculus of the mustached bat are tuned both to echo-delay and sound duration. Neuroreport 24:404-409

Masters WM, Raver KAS, Kazial KA (1995) Sonar signals of big brown bats, Eptesicus fuscus, contain information about individual identity, age and family affiliation. Anim Behav 50:1243-1260

Möhres FP (1967) Communicative characters of sonar signals in bats. Pp. 939-945. In: Busnel RG (ed) Animal sonar systems: biology and bionics, 2nd edn. Centre National de Recherches Zootechniques de Institut National de la Recherche Agronomique, Jouy-en-Josas, France, pp 719-1233

Neuweiler G (2003) Evolutionary aspects of bat echolocation. J Comp Physiol A 189:245-256

Neuweiler G, Metzner W, Heilmann U, Rübsamen R, Eckrich M, Costa HH (1987) Foraging behaviour and echolocation in the rufous horseshoe bat (Rhinolophus rouxi) of Sri Lanka. Behav Ecol Sociobiol 20:53-67

O'Neill WE, Suga N (1982) Encoding of target range and its representation in the auditory cortex of the mustached bat. J Neurosci 2:17-31

Racey PA (1988) Reproductive assessment in bats. In: Kunz TH (ed) Ecological and behavioral methods for the study of bats. Smithsonian Institution Press, Washington D.C., pp 31-45

Russo D, Jones G, Mucedda M (2001) Influence of age, sex and body size on echolocation calls of Mediterranean and Mehely's horseshoe bats, Rhinolophus euryale and R. mehelyi (Chiroptera: Rhinolophidae). Mammalia 65:429-436

Russo D, Mucedda M, Bello M, Biscardi S, Pidinchedda E, Jones G (2007) Divergent echolocation call frequencies in insular rhinolophids (Chiroptera): a case of character displacement? J Biogeogr 34:2129-2138

Schnitzler HU, Denzinger A (2011) Auditory fovea and Doppler shift compensation: adaptations for flutter detection in echolocating bats using CF-FM signals. J Comp Physiol A 197:541-559

Schnitzler HU, Henson OW Jr (1980) Performance of airborne animal sonar systems: I. Microchiroptera. In: Busnel RG, Fish JF (eds) Animal sonar systems. Plenum Press, New York, pp 109-181

Schuchmann M, Siemers BM (2010) Behavioral evidence for community-wide species discrimination from echolocation calls in bats. Am Nat 176:72-82

Siemers BM, Beedholm K, Dietz C, Dietz I, Ivanova T (2005) Is species identity, sex, age, or individual quality conveyed by echolocation call frequency in European horseshoe bats? Acta Chiropterol 7:259-274

Simmons JA, Vernon JA (1971) Echolocation: discrimination of targets by the bat, Eptesicus fuscus. J Exp Zool 176:315-328

Suga N (1990) Biosonar and neural computation in bats. Sci Am 262:60-68

Suga N, Niwa H, Taniguchi I, Margoliash D (1987) The personalized auditory cortex of the mustached bat: adaptation for echolocation. J Neurophysiol 58:643-654

Tang J, Fu ZY, Jen PHS, Chen QC (2011) Recovery cycles of single-on and double-on neurons in the inferior colliculus of the leaf-nosed bat, Hipposideros armiger. Brain Res 1385:114-126

Ulanovsky N, Moss CF (2008) What the bat's voice tells the bat's brain. Proc Natl Acad Sci U S A 105:8491-8498

Vanderelst D, Lee YF, Geipel I, Kalko EKV, Kuo YM, Peremans H (2013) The noseleaf of Rhinolophus formosae focuses the Frequency Modulated (FM) component of the calls. Front Physiol 4:191 
Voigt-Heucke SL, Taborsky M, Dechmann DNK (2010) A dual function of echolocation: bats use echolocation calls to identify familiar and unfamiliar individuals. Anim Behav 80:59-67

Wu CH, Jen PHS (2006) The role of GABAergic inhibition in shaping duration selectivity of bat inferior collicular neurons determined with temporally patterned sound trains. Hear Res 215:56-66

Wu CH, Jen PSH (2008) Echo frequency selectivity of duration-tuned inferior collicular neurons of the big brown bat, Eptesicus fuscus, determined with pulse-echo pairs. Neuroscience 156:1028-1038

Yovel Y, Melcon ML, Franz MO, Denzinger A, Schnitzler HU (2009) The voice of bats: how greater mouse-eared bats recognize individuals based on their echolocation calls. PLoS Comput Biol 5, e1000400

Zhang LB, Jones G, Zhang JS, Zhu GJ, Parsons S, Rossiter SJ, Zhang SY (2009) Recent surveys of bats (Mammalia: Chiroptera) from China. I. Rhinolophidae and Hipposideridae. Acta Chiropterol 11:71-88

Submit your manuscript to a SpringerOpen ${ }^{\circ}$ journal and benefit from:

- Convenient online submission

- Rigorous peer review

- Immediate publication on acceptance

- Open access: articles freely available online

- High visibility within the field

- Retaining the copyright to your article

Submit your next manuscript at $\gg$ springeropen.com 